\title{
Analysis of highway performance under mixed connected and regular vehicle environment
}

\author{
Zhao Zhang and Xianfeng (Terry) Yang \\ Department of Civil and Environmental Engineering, University of Utah, Salt Lake City, USA
}

\begin{abstract}
Purpose - This study aims to study the connected vehicle (CV) impact on highway operational performance under a mixed CV and regular vehicle (RV) environment.

Design/methodology/approach - The authors implemented a mixed traffic flow model, along with a CV speed control model, in the simulation environment. According to the different traffic characteristics between CVs and RVs, this research first analyzed how the operation of CVs can affect highway capacity under both one-lane and multi-lane cases. A hypothesis was then made that there shall exist a critical CV penetration rate that can significantly show the benefit of CV to the overall traffic. To prove this concept, this study simulated the mixed traffic pattern under various conditions.

Findings - The results of this research revealed that performing optimal speed control to CVs will concurrently benefit RVs by improving highway capacity. Furthermore, a critical CV penetration rate should exist at a specified traffic demand level, which can significantly reduce the speed difference between RVs and CVs. The results offer effective insight to understand the potential impacts of different CV penetration rates on highway operation performance.

Originality/value - This approach assumes that there shall exist a critical CV penetration rate that can maximize the benefits of CV implementations. CV penetration rate (the proportion of CVs in mixed traffic) is the key factor affecting the impacts of CV on freeway operational performance. The evaluation criteria for freeway operational performance are using average travel time under different given traffic demand patterns.
\end{abstract}

Keywords Connected vehicle, Highway capacity, Mixed traffic, Penetration rate, Variable speed limit

Paper type Research paper

\section{Introduction}

Connected vehicles (CV) are wireless connectivity-enabled vehicles that can communicate with their internal and external environments Lu et al. (2014). Recent advancements of information technologies enable CVs to communicate with each other $(\mathrm{V} 2 \mathrm{~V})$, roadside infrastructure (V2I) and the "Cloud" (V2C). Applications of CV technologies can offer great potentials of reshaping current concepts of planning, design and operation, which are expected to benefit transportation networks. For example, CV technology is considered as one of the most promising methods to improve traffic safety, which could dramatically reduce traffic crashes. According to the National Highway Traffic Safety Administration (NHTSA), 439,000-615,000 crashes will be prevented after the full implementation of V2V (National Highway Traffic Safety Administration [NHTSA], 2016). Such prediction is supported by a category of studies. For instance, Liu et al. (2017) adopted the Advanced Driver Assistance Systems for improving traffic safety under the CVs environment where vehicles are communicated with roadside infrastructure. Rahman and Abdel-Aty (2018) applied a vehicle

The current issue and full text archive of this journal is available on Emerald Insight at: https://www.emerald.com/insight/2399-9802.htm

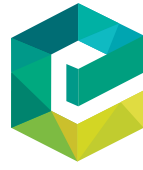

Journal of Intelligent and Connected Vehicles $4 / 2(2021) 68-79$

Emerald Publishing Limited [ISSN 2399-9802] [DOI 10.1108/JICV-10-2020-0011] behavior model for CV platoon based on the Intelligent Driver Model (IDM) model to evaluate the longitudinal safety of managed-lane CV platoons on expressways based on simulation results. Yang et al. (2017) developed simulationbased modeling and conducted an experimental study. Their results showed that $\mathrm{CV}$ s could be a viable way to reduce the risk of secondary crashes.

Besides the safety benefits, CV technologies can also help improve traffic efficiency and stability. Yang et al. (2020) demonstrated that eco-driving and platooning system supported by the advances of connected automated vehicle (CAV) technology can improve both fuel and operational efficiency of vehicles on the freeways. Chen et al. (2017) proposed a theoretical framework to evaluate how CV can change traffic capacity in equilibrium conditions, where shorter

(c) Zhao Zhang and Xianfeng (Terry) Yang. Published in fournal of Intelligent and Connected Vehicles. Published by Emerald Publishing Limited. This article is published under the Creative Commons Attribution (CC BY 4.0) licence. Anyone may reproduce, distribute, translate and create derivative works of this article (for both commercial and non-commercial purposes), subject to full attribution to the original publication and authors. The full terms of this licence maybe seen at http://creativecommons.org/licences/by/4.0/legalcode

This research is supported by MPC-590 - Impact of Connected Vehicle Technology on Traffic Safety under Different Highway Geometric Designs.

Received 29 October 2020

Revised 18 February 2021

20 May 2021

Accepted 24 June 2021 
gaps between CVs are believed to be the main contributor to highway capacity improvement. Talebpour and Mahmassani (2016) developed a framework that uses different models, with technology-appropriate assumptions, to simulate different vehicle types. Their study revealed that CVs can help improve string stability. Ghiasi et al. (2017) proposed an analytical stochastic formulation to analyze mixed traffic highway capacity, which is affected by $\mathrm{CV}$ penetration rate, $\mathrm{CV}$ platooning intensity and mixed traffic headway settings. Their results showed the benefits of $\mathrm{CV}$ technologies on improving traffic efficiency and were later implemented by (Han et al., 2017; Khondaker and Kattan, 2015; Zhang et al., 2018). On another regard, Wan et al. (2016) and Huang et al. (2018) analyzed the traffic pattern with mixed CVs and regular vehicles (RVs). They also derived optimization models to prove that CVs can help reduce fuel consumption. Furthermore, the effectiveness of CV platoon control has been investigated by Jia and Ngoduy (2016), Hu et al. (2020); Wang et al. (2020), Hao et al. (2020).

In summary, emerging $\mathrm{CV}$ technologies offer the potential to use real-time traffic information to improve traffic safety, efficiency and stability. Such benefits are usually achieved by optimal CV speed control, which is fulfilled by the communication between CVs and infrastructure (V2I). In the control loop, infrastructures provide advisory speed to CVs to follow, and CVs send their trajectory data and basic safety message to infrastructure for future feedback operations. In recent years, researchers started using the prediction model in traffic mixed with CVs and RVs. Han et al. (2017) developed variable speed limit (VSL) control strategies with $\mathrm{CV}$ technology to improve the discharge rate at recurrent freeway bottlenecks and to reduce system delay. Khondaker and Kattan (2015) proposed a VSL control algorithm with CV for simultaneously achieving mobility, safety and environmental improvements at the microscopic level. Hegyi et al. (2005) modified the METANET macroscopic traffic flow model with VSL control to determine the optimal speed. The effect of VSL was analyzed on aggregated traffic flow behaviors from a theoretical perspective by Papageorgiou et al. (2008) and Carlson et al. (2010). Their simulation results demonstrated that the total travel time is decreased by $15 \%$. Zhang et al. (2018) performed CV speed control on freeways with the sensitivity analysis of different $\mathrm{CV}$ penetration rate at the macroscopic level. Therefore, CV technologies, along with properly speed control algorithms, are approved as an effective way of benefiting traffic safety, efficiency and stability.

Notably, although CV technologies will soon go beyond testbeds and be implemented in real-world applications, it is expected that CVs and RVs will co-exist on the road for a long time. Hence, how to understand the interactions between CVs and RVs becomes a vital issue in the traffic control and operation domain. In addition, despite some existing studies proved the effectiveness of improving highway capacity performance by optimally controlling CVs, the penetration rate plays as a key role in affecting the system's performance. This is explicated by how RV drivers would respond to the speed change of their nearby CVs. Grounded on preliminary simulation studies, it can be observed that RVs will follow CVs in the traffic flow when they have no sufficient gap to make lane-changing (e.g. under congested condition or with a high
$\mathrm{CV}$ penetration rate). In such cases, the speed control of CVs will greatly affect RVs' speeds in the meantime. However, under the conditions of light traffic or low CV rates, RVs can easily change lanes to avoid following CVs. The CVs are advised to slow down, it may even increase the potential crash rate due to increased lane-changing maneuvers. Therefore, given a congestion level, there should exist a critical CV penetration rate that can fully demonstrate the benefit of $\mathrm{CV}$ impact.

Grounded on our developed macroscopic mixed traffic flow and CV speed optimization model (Zhang et al., 2018), this paper intends to explore the existence of critical CV penetration rate and study its impact to the freeway operational performance. More specifically, this study analyzes the freeway traffic flow characteristics under a mixed $\mathrm{CV}$ and $\mathrm{RV}$ environment. To understand the $\mathrm{CV}$ impact to the overall traffic flow, this study simulates the mixed traffic pattern with different $\mathrm{CV}$ penetration rates and various traffic demands levels. Considering the different driving characteristics of CVs and RVs, our research firstly analyzes how the implementation of CVs can affect highway capacity under both one-lane and multi-lane cases. The simulation results reveal that performing optimal speed control to CVs will concurrently benefit RVs due to their interactions in the mixed traffic streams. The highway capacity will consequently be increased. Also, given a demand pattern on a target freeway segment, it exists a critical CV penetration rate that can significantly reduce the speed difference between RVs and CVs. With the increase of traffic demand volumes, such a critical $\mathrm{CV}$ rate intends to be a smaller value. Those results could offer effective insight for traffic operators to better understand the potential impacts of different $\mathrm{CV}$ penetration rates on highway capacity.

The rest of the paper is organized as follows: Section 2 will discuss the research background and illustrate the problem of nature to be solved; following sections offer an overview of the control model and extensive simulation studies to identify the critical CV penetration rates under various scenarios; research findings, conclusions and future research direction are then summarized in the last section.

\section{Highway capacity under connected vehicles impact}

In the literature, many recent research efforts have been attracted to CV-related optimal control. Meanwhile, a similar concept but for RVs only, named VSL control, has also been studied extensively in the literature. By dynamically changing the displayed speed limit, VSL is initially developed to reduce the rear-end collisions and improve traffic safety by decreasing the speed difference on freeway segments (Steel et al., 2005; Ulfarsson et al., 2005). Later researches have further explored its potential for improving traffic efficiency and stability, and both reactive and proactive control models are derived (Kang et al., 2004; Kwon et al., 2007; Abdel-Aty et al., 2006; Abdel-Aty et al., 2008; Yang et al., 2017; Lyu et al., 2017). Different from VSL control, which typically assumes homogenous vehicles in the traffic, CV-based control models are usually operated under mixed traffic patterns. Hence, how RVs will react to the speed change of CVs becomes a vital issue that is worthy of exploration. For proof-of-concept, our research team has 
conducted a simulation study at an intersection to investigate the impact of $\mathrm{CV}$ control on the traffic stream.

Figure 1 shows the vehicle speed distribution under two simulation cases. Case 1 was simulated with mixed traffic containing both CVs and RVs, while Case 2 was simulated with RVs only. In case 1, optimal speed control is performed on CVs to smooth traffic speed transition from the upstream high-speed segment to the downstream low-speed segment. The result comparisons indicate that despite speed control is only performed on CVs, the average speed of RVs would also be reduced as they are sharing the road. However, the speed difference between CVs and RVs can still be observed. Hence, a conclusion can be drawn that speed control of CVs can impact the speed of nearby RVs. However, the significance of such impact may subject to the congestion level and CV penetration rate. In this study, our research team would conduct further research to analyze this critical issue, named CV impact.

According to existing studies, the application of $\mathrm{CV}$ technologies can improve highway capacity and efficiency (Chen et al. (2017); Abdulsattar et al., 2020; Ghiasi et al., 2017). Chen et al. (2017) conducted a comprehensive study to show how CV improve highway capacity and efficiency at a macroscopic level. They found that capacity related to $\mathrm{CV}$ penetration rate, platoon size, inter-vehicle spacing and lane policies and traffic mixed with CVs and RVs can lead to higher capacity and strict segregation of CVs and RVs will bring lower capacity. Based on the extended analysis, they get the conclusion that CVs use the most efficient lanes on highway lead to make the best use of roadway efficiency and gain the highest capacity. On the contrary, Ghiasi et al. (2017) came the opposite conclusion, based on the analytical stochastic formulation for mixed traffic highway capacity, higher $\mathrm{CV}$ penetration rates and platooning intensities not always improve highway capacity. Therefore, the CV penetration rate (Chen et al., 2017; Ghiasi et al., 2017; Bansal and Kockelman, 2017; Chen et al., 2016; Lavasani et al., 2016) need to be further analyzed to understand how it can impact on highway capacity and efficiency.

For the convenience of discussion, this study selects a single-lane highway and a two-lane highway for study. For a single-lane highway segment, its capacity can be estimated by the inter-vehicle spacing. Many existing

\section{Figure 1 Time-dependent speed distribution of vehicles}

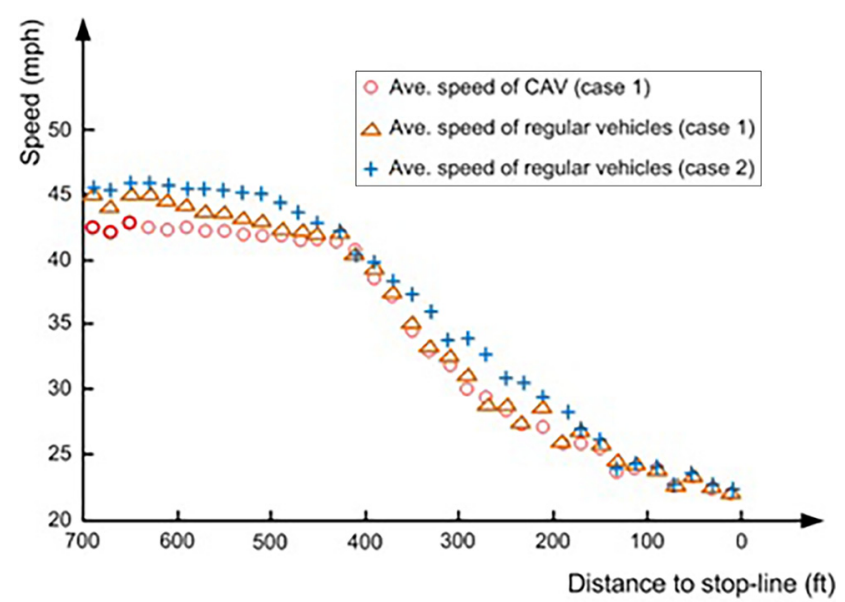

studies (Ahn et al., 2004; Newell, 2002) defined this spacing is the distance between the reference points of an immediate follower and its leader, such as from the front bumper to the front bumper. The spacing is shown in Figure 2, $\mathrm{S}_{0}$ denotes the spacing between RVs; $\mathrm{S}_{1}$ indicates the spacing between $\mathrm{RV}$ and $\mathrm{CV}$ ( $\mathrm{CV}$ ahead); $\mathrm{S}_{2}$ denotes the spacing between $\mathrm{CV}$; and $\mathrm{S}_{3}$ denotes the spacing between $\mathrm{CV}$ and RV (RV ahead). By assuming both CVs and RVs drive at a constant free-flow speed and based on the previous study finished by Chen et al. (2017), the relations among all types of spacings can express as $S_{2}<S_{0}$ $<\mathrm{S}_{3}<\mathrm{S}_{1}$. It means the $\mathrm{CV}$ can save inter-vehicle spacing, which further indicates that $\mathrm{CV}$ can help improve roadway capacity.

Such phenomenon can be further proved in the fundamental diagram, as shown in Figure 3, where $\mathrm{C}_{0}$ denotes lane capacity with RVs only; $\mathrm{C}_{1}$ denotes lane capacity with RVs and $\mathrm{CVs} ; q$ denotes the traffic flow; $u_{0}$ denotes the free-flow speed until reaching critical capacity with RVs only; $u_{1}$ denotes the free-flow speed until reaching critical capacity with RVs and CVs; $w_{0}$ denotes the freeflow speed until reaching congested with RVs only; $w_{1}$ denotes the free-flow speed until reaching congested with RVs and CVs; and $d$ denotes the corresponding density under these two different traffic environment. With reduced vehicle spacing, it can be observed in Figure 3 that the highway capacity with mixed with CVs and RVs is higher than the case with RVs only.

For a two-lane highway segment, the capacity estimation under $\mathrm{CV}$ impact becomes more complicated and is subject to many factors. However, on a multi-lane highway segment, RVs can choose to make lane-changing when it follows a slow CV. Figure 4(a) illustrates a case that CVs are traveling at a lower speed compared with RVs. Due to the light traffic condition, RVs can easily pass CVs by making a few lane-changing maneuvers. Hence, the impact of CV speed control on the entire traffic flow is quite minimal in such cases. In contrast, Figure 4(b) shows a congested highway segment where RVs have to follow CVs due to the difficulty of making lanechanging. Under such conditions, it can be expected that CVs' speed control can greatly affect the entire traffic.

From the microscopic level, moving of traffic streams can be characterized by both car-following models and lanechanging models. Under the mixed $\mathrm{CV}$ and RV pattern, car-following behaviors can be categorized as four categories: $\mathrm{CV}$ follows $\mathrm{CV}, \mathrm{CV}$ follows $\mathrm{RV}, \mathrm{RV}$ follows $\mathrm{CV}$, and RV follows RV. Similar to the case of the one-lane highway, the main variation of these four following scenarios is the different minimum following spacing. For modeling the discretionary lane-changing behaviors, a probabilistic form has been widely used in existing simulation tools, where the probability is subject to the lane speed variations and the number of safe lane-changing gaps. Applying the same logic to analyze the mixed CV and RV traffic pattern, it can be concluded that more RV lane-changing behaviors may be observed if CVs are operated at lower speeds; and the number of safe lanechanging gaps is sufficient. Therefore, given the CV optimal speed control pattern and highway traffic demand profile, there shall exist a critical $\mathrm{CV}$ penetration rate that can maximize the $\mathrm{CV}$ impact on the traffic flow without increasing lane-changing significantly. 
Figure 2 Inter-vehicle spacing in traffic mixed with CV and RV

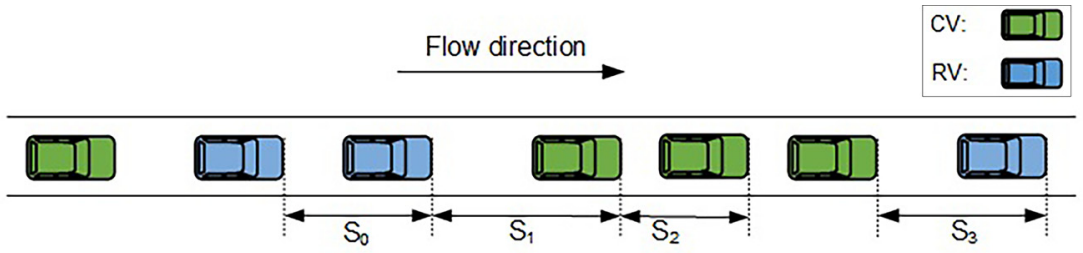

Figure 3 Fundamental diagram with RV only and mixed with CV and RV

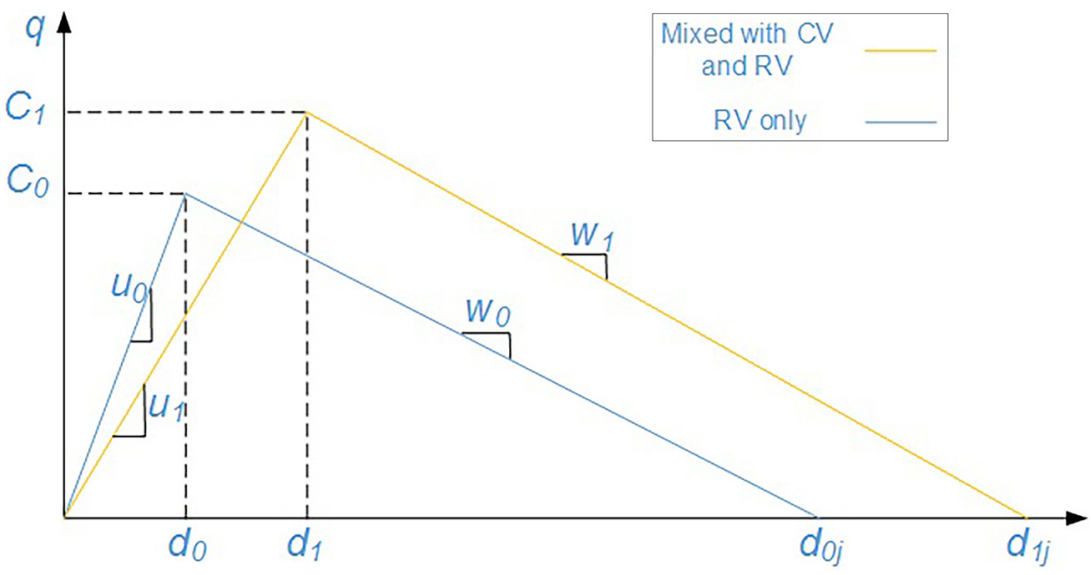

Figure 4 (a) Light traffic with CVs (b) congested traffic with CVs

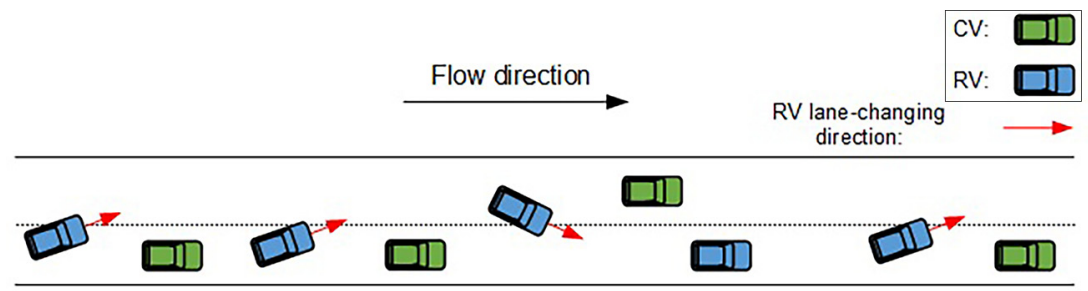

(a)

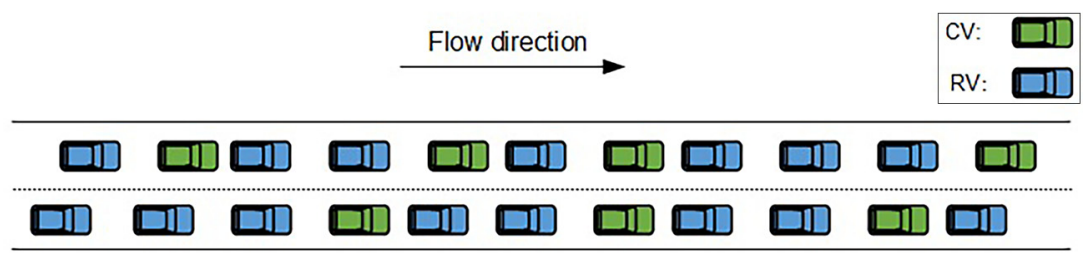

(b)

\section{Macroscopic mixed traffic flow with cv optimization model}

To analyze the impact of CV optimal speed control on the mixed traffic pattern, this study implements a macroscopic mixed traffic flow with CV optimization model developed by Zhang et al. (2018), which consists of two main functions: macroscopic traffic state estimation and $\mathrm{CV}$ speed optimization.

\subsection{Macroscopic mixed traffic state estimation}

Under mixed $\mathrm{CV}$ and RV traffic conditions, one of the most challenging issues in traffic state estimation is how to capture the interactions between $\mathrm{CVs}$ and RVs. For example, V2I communication platform can facilitate the information change between vehicles and infrastructures. From the operation aspect, V2I will provide enriched traffic data such as CV trajectories to support the real-time control needs. On the other regards, the control module can send advisory speeds to the CVs for the 
benefit of improving freeway operational efficiency. However, such CV speed control will impact nearby RVs as they are sharing the roads. Then a vital question raised here is how RVs will response to the speed change of CVs. Based on the simulations conducted in the literature, it can be expected that RVs' behaviors would be subject to the traffic congestion level and $\mathrm{CV}$ penetration rates.

To model the mixed traffic flow pattern, this study is based on a macroscopic traffic flow model proposed by Papageorgiou et al. (1990) that can function to predict future traffic state (speeds, flow and density). However, such a conventional traffic flow model may fall short of effectiveness as speed control is placed on CVs because it analyzed all vehicles as a group in each freeway subsegment. Meanwhile, operation of CVs would affect RVs' speeds because they are sharing the road. To overcome such issues, Yang et al. (2020) extended the macroscopic model by dividing vehicles into two different classes. Assume the case of two vehicle classes (e.g. CVs and RVs) without loss of generality, the variation of traffic characteristic between different classes of vehicles can be described as follows:

$d_{j, i}(k+1)=d_{j, i}(k)+\frac{\Delta T}{L_{i} \lambda_{i}}\left[q_{j, i, i n}(k)-q_{j, i, \text { out }}(k)+r_{j, i}(k)-s_{j, i}(k)\right]$

where $d_{j, i}(k)$ denotes the density of class $j$ vehicles for segment $i$ at time step $k ; \Delta T$ denotes the length of update time interval; $L_{i}$ denotes the length of segment $i ; \lambda_{i}$ denotes the number of lanes of segment $i ; q_{j, i, i n}(k)$ denotes the flow rate of class $j$ vehicles entering segment $i$ from upstream segment $i$ between steps $(k, k+1) ; q_{j, i}$, ${ }_{\text {out }}(k)$ denotes the flow rate of class $j$ vehicles leaving segment $i$ to downstream segment $i$ between steps $(k, k+1) ; r_{j, i}(k)$ denote the flow rate of class $j$ vehicles entering segment $i$ from on-ramps between steps $(k, k+1) ; s_{j, i}(k)$ denotes the flow rate of class $j$ vehicles leaving segment $i$ from off-ramps between steps $(k, k+1)$.

And the flow transition between adjacent segments is as follows:

$$
\begin{aligned}
& q_{j, i, \text { in }}(k)=q_{j, i-1, \text { out }}(k) \\
& q_{j, i, \text { in }}(k)=q_{j, i-1, \text { out }}(k)
\end{aligned}
$$

By defining:

$$
V_{j, i}\left[d_{j, i}(k)\right]=u_{f, j i} \exp \left[-\frac{1}{a_{j i}}\left(\frac{d_{1, i}(k)+d_{2, i}(k)}{d_{c r, j i}}\right)^{a_{j i}}\right]
$$

where $u_{f, j i}$ denotes the free-flow speed of segment $i$ for class $j$ vehicles; $a_{, j i}$ denotes the speed exponent term of segment $i$ for class $j$ vehicles; $d_{c r, j i}$ denotes the critical density of segment $i$ for class $j$ vehicles.

The mean speed of RVs within each segment can be estimated by:

$$
\begin{aligned}
u_{1, i}(k+1)= & u(k)+\frac{\Delta T}{\tau_{i}}+\frac{\Delta T}{L_{i}} u_{1, i}(k)\left[u_{1, i-1}(k)-u_{1, i}(k)\right] \\
& -\frac{\gamma_{i} \Delta T}{\tau_{i} L_{i}} \frac{\left[d_{1, i+1}(k)+d_{2, i+1}(k)-d_{1, i}(k)-d_{2, i}(k)\right]}{\left[d_{1, i}(k)+d_{2, i}(k)\right]} \\
& -\frac{\beta_{i} \Delta T}{\tau_{i}}\left[V_{1, i}(k)-V_{2, i}(k)\right]
\end{aligned}
$$

Similarly, the mean speed of CVs can be obtained with:

$$
\begin{aligned}
u_{2, i}(k+1)= & u_{2, i}(k)+\frac{\Delta T}{\tau_{i}}\left[V_{2, i}(k)-u_{2, i}(k)\right] \\
& +\frac{\Delta T}{L_{i}} u_{2, i}(k)\left[u_{2, i-1}(k)-u_{2, i}(k)\right] \\
& -\frac{\gamma_{i} \Delta T}{\tau_{i} L_{i}} \frac{\left[d_{1, i+1}(k)+d_{2, i+1}(k)-d_{1, i}(k)-d_{2, i}(k)\right]}{\left[d_{1, i}(k)+d_{2, i}(k)\right]}
\end{aligned}
$$

where $u_{j, i}(k)$ denotes the mean speed of class $j$ vehicles for segment $i$ at time step $\mathrm{k} ; V_{j}\left[d_{j, i}(k)\right]$ denotes the static speed of class $j$ vehicles for segment $i$ at time $\mathrm{k}$ with respect to the density of class $j$ vehicles; $\tau_{i}, \gamma_{i}, \kappa_{i}, \beta_{i}$ denotes the parameters in the dynamic speed equations of segment $i$. And the following equations hold:

$$
q_{j, i}(k)=\lambda_{i} d_{j, i}(k) u_{j, i}(k)
$$

Notably, the interaction between RVs and CVs is represented by the speed difference between CVs and RVs. In equation (5), a set of parameters $\beta_{i}$ was introduced into the term to reflect how CVs' speed will impact RVs' speed. Then the flowing equations are used for restricting equation (6) to represent the flow of $\mathrm{CV}$ and RV on the segment with V2I communication:

$$
\begin{gathered}
q_{1, i, \text { in }}(k)=\left(1-\alpha_{i}\right)\left[q_{1, i-1, \text { out }}(k)+q_{2, i-1, \text { out }}(k)\right] \\
q_{2, i, \text { in }}(k)=\alpha_{i}\left[q_{1, i-1, \text { out }}(k)+q_{2, i-1, \text { out }}(k)\right]
\end{gathered}
$$

where, $\alpha_{i}$ denotes the penetration rate of $\mathrm{CV}$.

\subsection{Connected vehicles optimization model}

Grounded on the macroscopic mixed traffic state estimation model, an optimization function will provide optimal advisory speeds to CVs via V2I communications. The embedded optimization model has the following objective of minimizing the function of total travel time over the controlled segment:

$$
\min \sum \sum \lambda_{i}\left[d_{1, i}(k)+d_{2, i}(k)\right] \Delta T
$$

For implementing the optimization model and select the proper control speed for the projected control period, the following constraints should be placed:

$\begin{cases}u_{j a m} \leq u_{j, i}(k) \leq u_{f, j i}, & \text { segment } i \text { without coverage of } V 2 I \\ u_{j a m} \leq u_{j, i}(k) \leq u_{f, j i} v_{i}(k), & \text { segment } i \text { with V2I communications }\end{cases}$

$$
\begin{gathered}
0<v_{i}(k) \leq 1 \\
0 \leq d_{j, i} \leq d_{j a m} \\
-\delta \leq u_{f, j i} v_{i}(k)-u_{f, j i} v_{i}(k-1) \leq \delta
\end{gathered}
$$

where $\delta$ denotes that maximum speed variation of $\mathrm{CVs}$ between two consecutive time slots. 


\section{Freeway performance study}

\subsection{Simulation network}

This study selects a typical two-lane freeway as an example for analysis. As shown in Figure 5, the freeway stretch is divided into eight segments, and the total length is $4 \mathrm{~km}$. There is one on-ramp on $3^{\text {rd }}$ segment and one off-ramp on 5 th segment. Then the macroscopic traffic flow model is implemented to simulate the mixed traffic flow pattern when CVs follow the optimal advisory speeds. The model parameters are set up based on the existing study finished by Wang and Papageorgiou (2008). $\tau, \nu, \kappa, \delta, u_{f}, d_{c r}$ a is set to $20 \mathrm{~s}, 35 \mathrm{~km}^{2} / \mathrm{h}, 13 \mathrm{veh} / \mathrm{km} / \mathrm{lane}$, $1.4,120 \mathrm{~km} / \mathrm{h}, 33.5 \mathrm{veh} / \mathrm{km}$ and 1.4324 , respectively.

To fully study the CV impact on the RVs, this study has simulated the mixed traffic flow under various traffic patterns, as shown in Table 1 . The $1,500 \mathrm{veh} / \mathrm{h}$ is set up for mimicking the free flow environment and $4,000 \mathrm{veh} / \mathrm{h}$ is set up for simulating the congestion environment. Between these two traffic demand conditions, we set 500 increments as a new case to learn the CV impact in the traffic stream. Furthermore, under a given traffic demand pattern, we set $5 \%$ increments of $\mathrm{CV}$ penetration rate between $5 \%$ and $80 \%$ to learn the CV impact in the traffic stream.

In equation (5), CV speed impact on RV groups is represented by the key factor $\beta_{i}$. Notably, the value of the factor shall be subject to both demand levels and CV ratios. Follow the similar procedure of existing study Bansal and Kockelman (2017), $\beta_{i}$ is calibrated with microscopic simulations and the corresponding value within different simulation scenarios is shown in Figure 6.

\subsection{Performance index}

To evaluate the effectiveness of the proposed model, average travel time (TT) under different given traffic demand patterns is used to analyze the simulation results.

$$
T T=\frac{\sum_{i=1}^{n} \frac{L}{u_{i}(k)}}{n}
$$

where $L$ denotes the length of each freeway subsegment; $u_{i}(k)$ denotes the mean speed of segment $i$ at time step k; $n$ denotes the number of subsegment.

\subsection{Connected vehicles impact analysis}

Selecting average travel time of all vehicles along the studied freeway segment as the performance index, this study has adopted the Monte Carlo simulation technique to evaluate the freeway performance. All designed scenarios are shown in Table 1, and the results are summarized in Figure 7. By
Table 1 Mixed traffic flow simulation scenarios

\begin{tabular}{lll}
\hline $\begin{array}{l}\text { Demand level } \\
\text { (two lanes) }\end{array}$ & CV penetration rate & $\begin{array}{l}\text { CV flows } \\
\text { (two lanes) }\end{array}$ \\
\hline 1,500 veh $/ \mathrm{h}$ & $5 \%-80 \%$ with 5\% increment & $75-1,200 \mathrm{veh} / \mathrm{hr}$ \\
$\mathbf{2 , 0 0 0} \mathrm{veh} / \mathrm{h}$ & $5 \%-80 \%$ with 5\% increment & $100-1,600 \mathrm{veh} / \mathrm{hr}$ \\
$\mathbf{2 , 5 0 0} \mathrm{veh} / \mathrm{h}$ & $5 \%-80 \%$ with 5\% increment & $125-2,000 \mathrm{veh} / \mathrm{hr}$ \\
$\mathbf{3 , 0 0 0} \mathrm{veh} / \mathrm{h}$ & $5 \%-80 \%$ with 5\% increment & $150-2,400 \mathrm{veh} / \mathrm{hr}$ \\
$\mathbf{3 , 5 0 0} \mathrm{veh} / \mathrm{h}$ & $5 \%-80 \%$ with 5\% increment & $175-2,800 \mathrm{veh} / \mathrm{hr}$ \\
$\mathbf{4 , 0 0 0} \mathrm{veh} / \mathrm{h}$ & $5 \%-80 \%$ with 5\% increment & $200-3,200 \mathrm{veh} / \mathrm{hr}$
\end{tabular}

comparing the cases of different $\mathrm{CV}$ penetration rates, it can be observed that the average travel time will be reduced with the increasing of $\mathrm{CV}$ penetration rate, which proves the benefit of implementing CV technology. Notably, at the low demand level, such benefit is not significant. For example, with the $1,500 \mathrm{veh} / \mathrm{h}$ traffic demand, the corresponding travel times of $5 \%$ and $80 \% \mathrm{CV}$ ratios are obtained as $129.1 \mathrm{~s}$ and $121.4 \mathrm{~s}$, respectively. In the contrast, in the cases with $4,000 \mathrm{veh} / \mathrm{h}$ upstream arriving flow, the travel time difference by these two $\mathrm{CV}$ ratios is $18 \mathrm{~s}$. Hence, under a more congested traffic environment, the optimal CV speed control system can yield more benefits on reducing vehicle delays. However, it shall be noted that all scenarios are simulated at the under-saturated condition. When traffic demand exceeds freeway capacity, the operational benefits by CVs may be minimized.

To study the CV impact on RVs and understand their interactions, this research also compared the travel time performance of four different vehicle groups:

1 all vehicles without $\mathrm{CV}$ involved;

2 all vehicles with CV optimal control;

3 CVs under optimal speed control; and

4 RVs under CV optimal control.

Figure 8 presents the resulting average travel time under different $\mathrm{CV}$ rates and demand levels. By comparing the cases under $\mathrm{CV}$ control with the ones without CVs, it can be noted that providing advisory speeds to CVs can help reduce the travel time of the entire traffic stream. Also, CVs experienced much lower travel time compared with RVs in all scenarios. With the increasing $\mathrm{CV}$ penetration rate, the reduction of $\mathrm{CV}$ travel time is quite minimal under low demand conditions. With higher upstream traffic flow, the optimal CV speed harmonization will yield more travel time reduction when $\mathrm{CV}$ ratio increased. In addition, a further performance comparison between $\mathrm{CV}$ and $\mathrm{RV}$ groups revealed that the CV impact, indicated by RV travel time reduction along with $\mathrm{CV}$ optimal control, becomes more significant when the system involving a

Figure 5 Stretch of the studied freeway

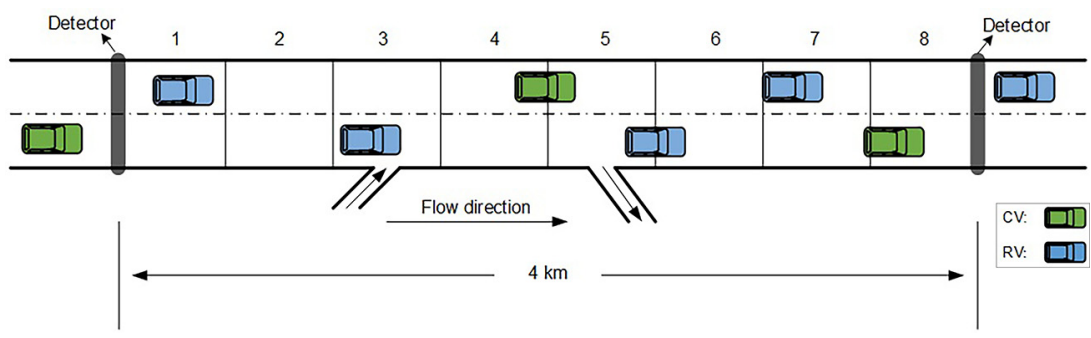


Figure 6 The distribution of in different scenarios

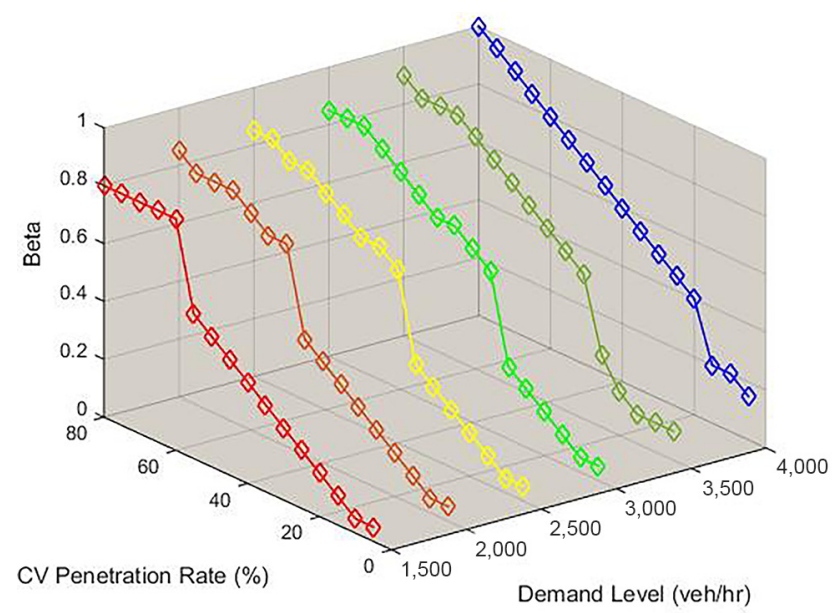

larger portion of CVs. There is a critical CV penetration rate under each level of demand that can greatly reduce the speed difference between CVs and RVs. Based on Figure 8 (a)-(f), the critical CV rates under various demand patterns and corresponding average travel time of different vehicle groups are summarized in Table 2.

To better illustrate the existence of critical CV penetration rate and demonstrate how it can impact highway performance, Figure 9 summarizes the reduced average travel time of all vehicles under different scenarios, compared with the cases of no CV control. Notably, under each level of demand, the travel time reduction suddenly becomes significant after the $\mathrm{CV}$ ratio reached the critical value. After passing the critical $\mathrm{CV}$ rates, the average travel time will keep reducing with the increase of $\mathrm{CV}$ rates. However, under the low demand scenarios, such travel time reduction is not significant.

To further analyze the CV speed impact on RVs under critical CV rate conditions, Figure 10 (a)-(f) presents the time- dependent travel time of all traffic, CVs and RVs. In each case, the travel time of all traffic generated the highest travel time when $\mathrm{CV}$ optimal control is unavailable, on the contrary, the travel time of $\mathrm{CV}$ obtained the lowest travel time because the CVs with optimal speed control. The travel time of all traffic under control is dramatically decreased because optimal speed control is performed on CVs within the mixed $\mathrm{CV}$ and RV traffic pattern. It is indicated that $\mathrm{CV}$ optimal control can help reduce the travel time of both CVs and RVs. Also, RVs have very similar travel time compared with CVs. Hence, one can conclude that RVs were very likely to follow CVs within the traffic flow when $\mathrm{CV}$ rate reached a critical value under each level of demand.

In summary, we can summarize the following key findings based on the comprehensive simulation analysis:

- within the mixed CV and RV traffic pattern, performing optimal speed control to CVs will concurrently benefit RVs because they are sharing the roadway;

- given a demand pattern on a target freeway segment, there should exist a critical CV penetration rate that can greatly improve the performance of CV-based control systems;

- once the CV penetration rate reached the critical value, the speed difference between RVs and CVs will become insignificant;

- mixed traffic under high demand level (but still below the capacity) intends to have smaller critical $\mathrm{CV}$ rate compared with the case under low demand level.

Notably, although this study has used numerical tests to show the existence of a critical CV penetration rate and indicated its value was subject to demand levels, it is essential to complete theoretical proof in future studies. Also, more simulation and field studies shall be conducted to provide guidance on how to determine the critical $\mathrm{CV}$ ratio to maximize the highway capacity.

\section{Discussion}

This study demonstrated that the CV impact on highway operational performance by a developed macroscopic mixed

Figure 7 Freeway average travel time under different scenarios

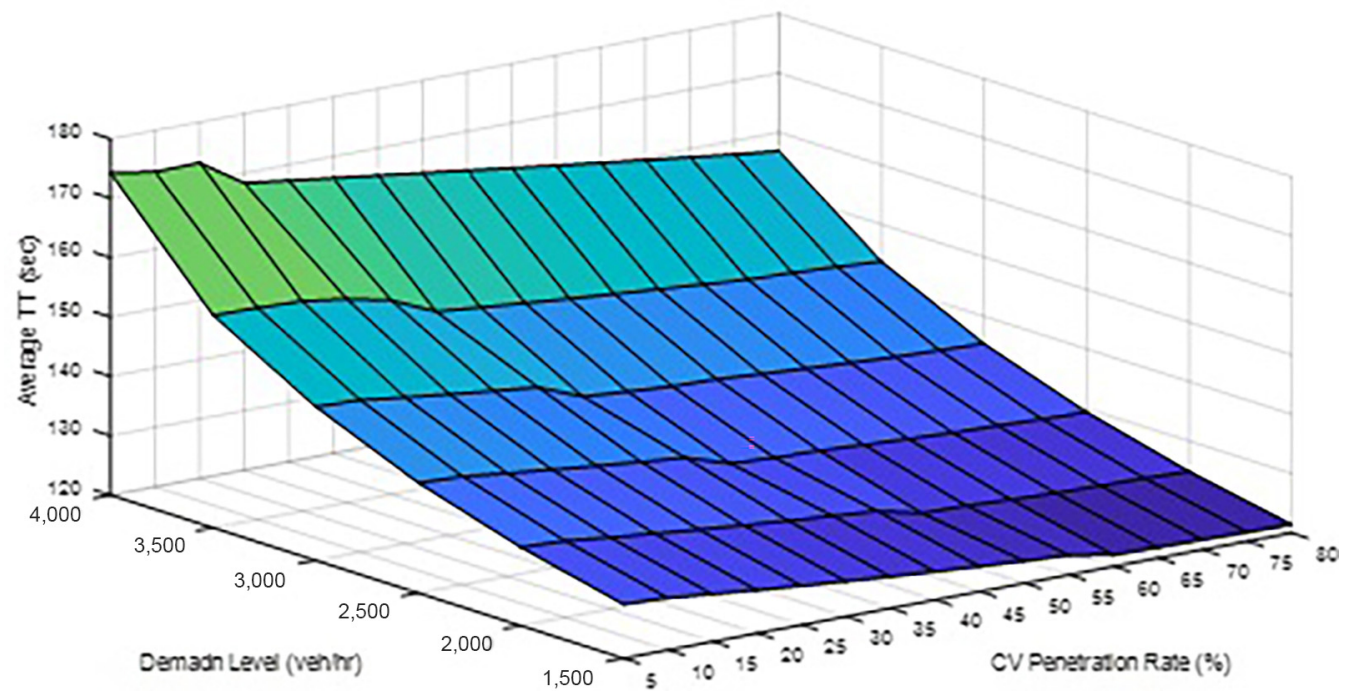


Figure 8 Average travel time comparison between different vehicle groups

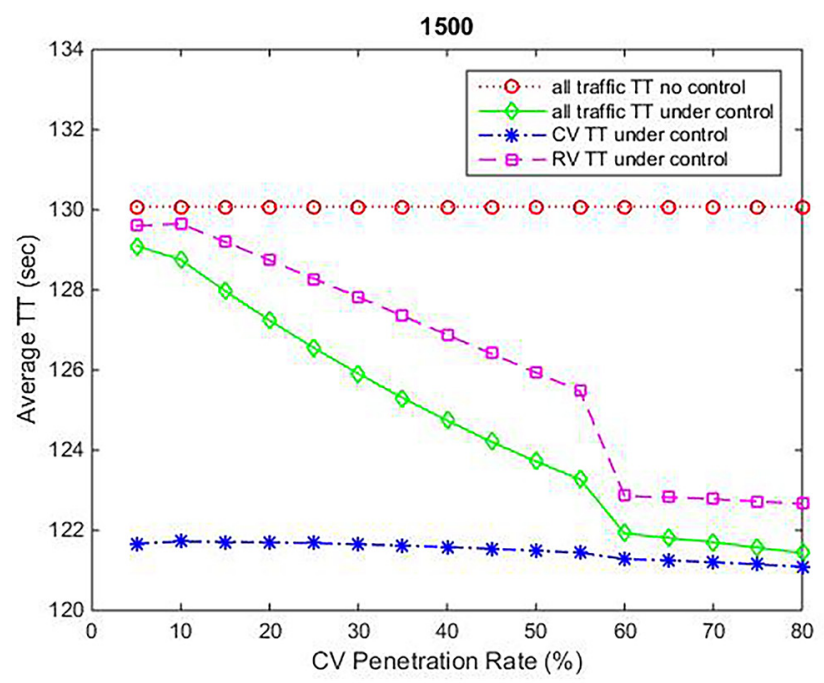

(a)

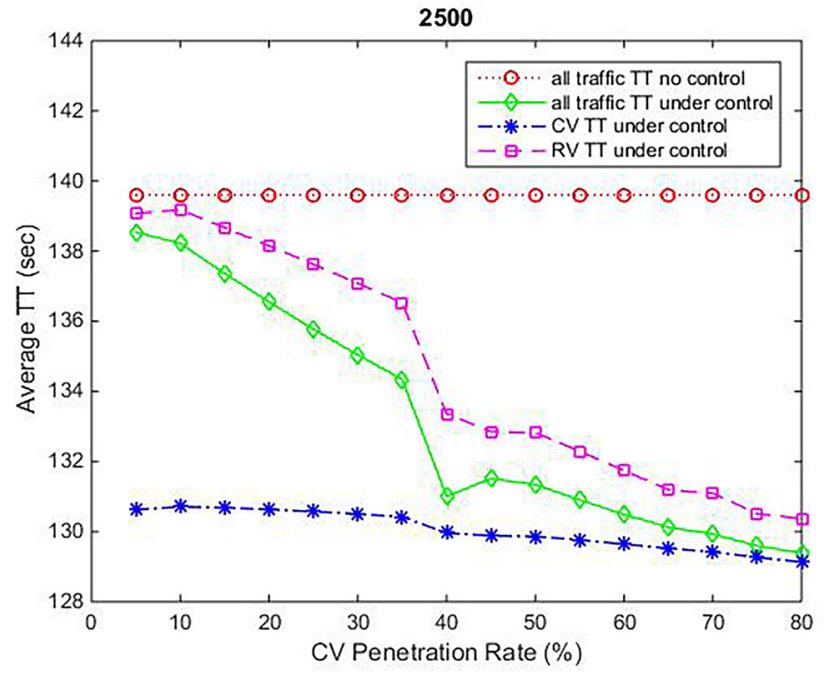

(c)

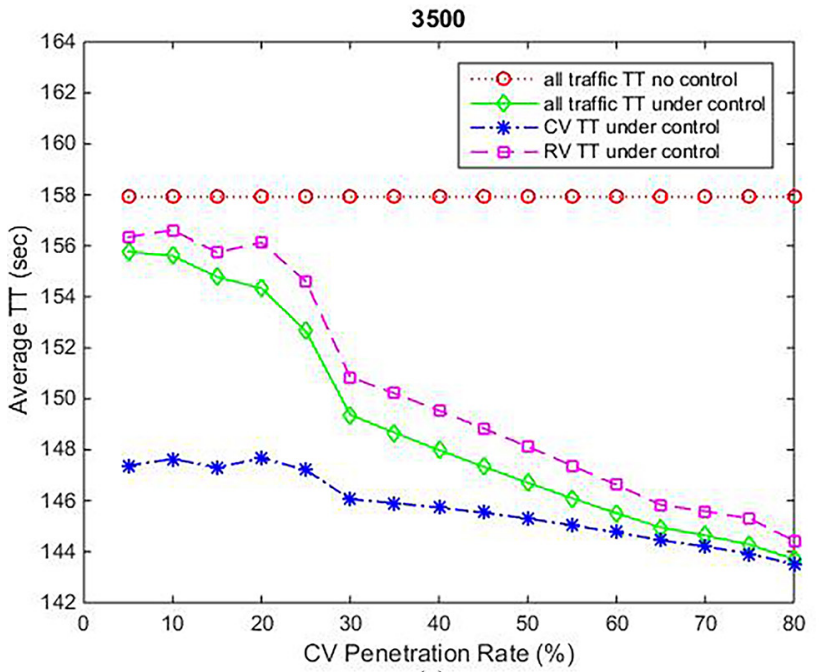

(e)

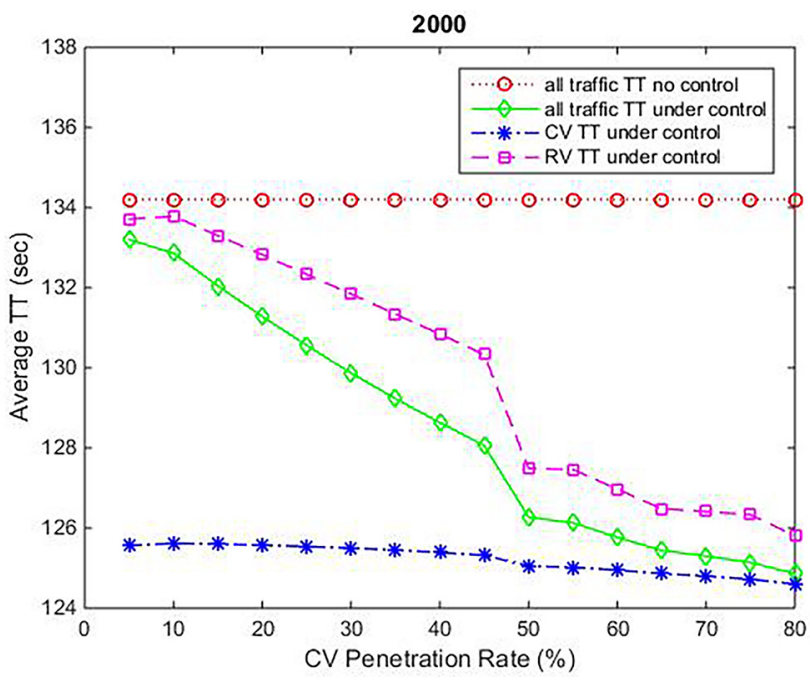

(b)

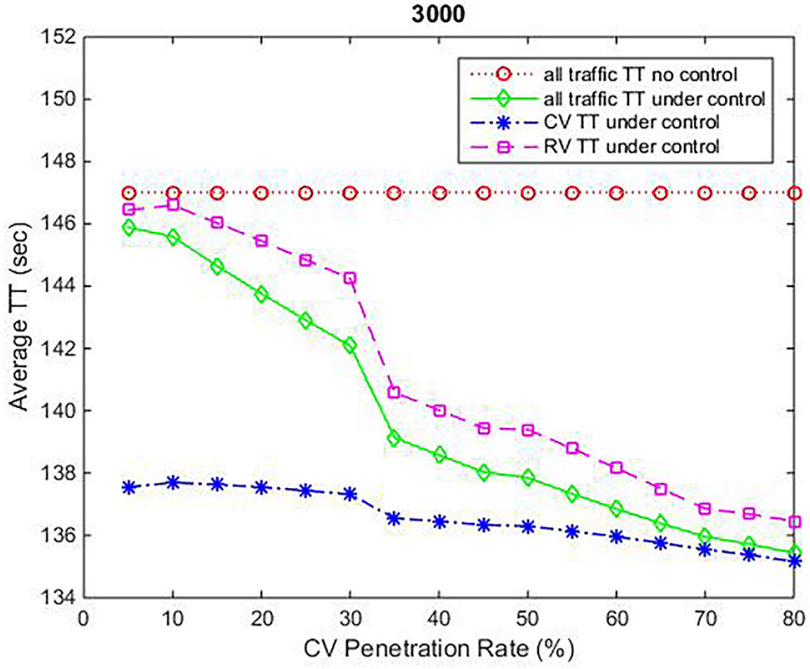

(d)

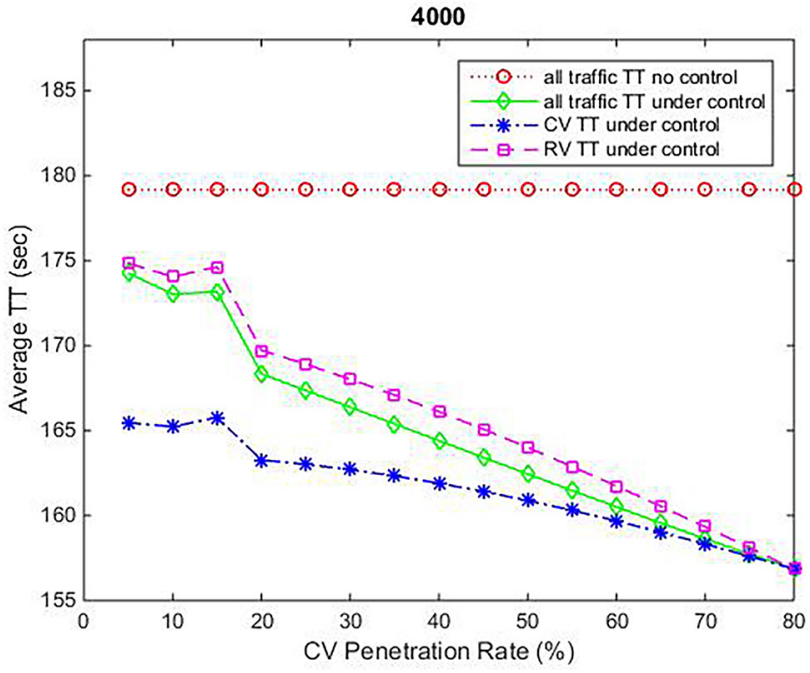

(f) 
Table 2 The simulation results of the scenarios with critical cv ratio

\begin{tabular}{lccccc}
\hline Demand level & $\mathbf{1 , 5 0 0}$ & $\mathbf{2 , 0 0 0}$ & $\mathbf{2 , 5 0 0}$ & $\mathbf{3 , 0 0 0}$ & $\mathbf{3 , 5 0 0}$ \\
\hline Critical CV ratio (\%) & 60 & 50 & 40 & 35 & $\mathbf{4 , 0 0 0}$ \\
All traffic TT with no CV & 130.08 & 134.20 & 139.60 & 147.01 & 157.92 \\
All traffic TT with CV & 121.92 & 126.27 & 131.98 & 139.14 & 149.37 \\
TT of CV & 121.28 & 125.05 & 129.96 & 136.55 & 146.06 \\
TT of RV & 122.86 & 127.49 & 133.36 & 140.59 & 150.85 \\
TT difference between CV and RV (\%) & 1.3 & 2.4 & 3.4 & 4 & 163.27 \\
\hline
\end{tabular}

Figure 9 Reduced average travel time under different scenarios and critical CV rates

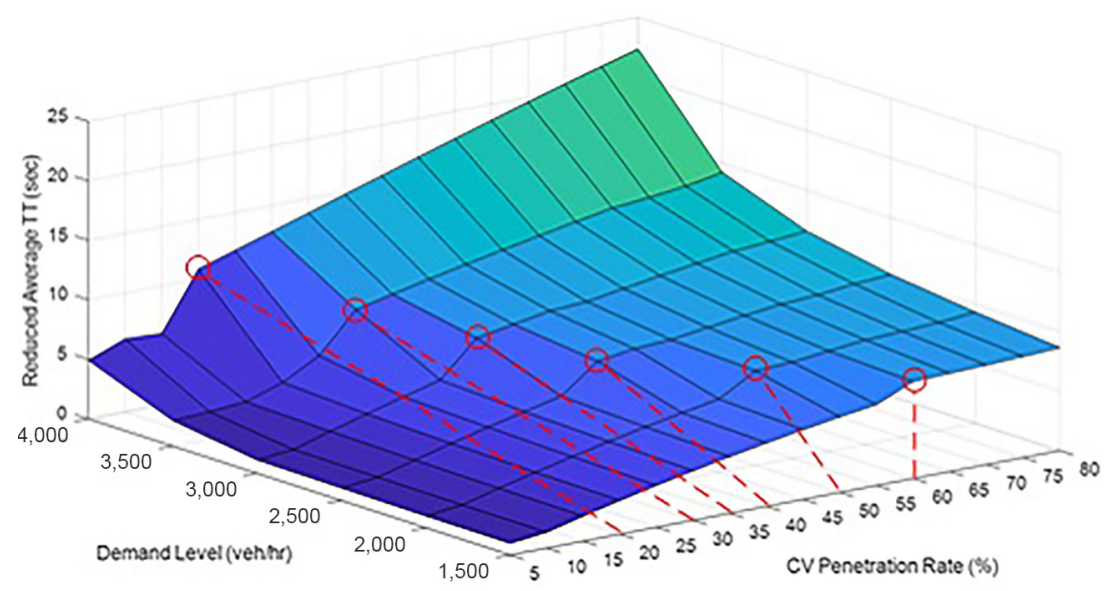

traffic flow with CV optimization model under mixed traffic with CVs and RVs simulation environment. The study results indicate that there should exist a critical $\mathrm{CV}$ penetration rate that can greatly improve highway operational performance. The macroscopic mixed traffic flow with $\mathrm{CV}$ optimization model is developed based on the traditional traffic flow model developed by Papageorgiou et al. (2008). To make the developed model could simulate the CV impact on the traffic flow and easy to implement, the influence of the CVs is added to the model with an adjustable factor $\beta \mathrm{i}$. The most significant improvement of this model due to more simulation function can be achieved. However, there are still a few limitations that need to be overcome.

\subsection{Microscopic influence between connected vehicles and regular vehicles}

This research study the influence between CV and RV with different $\mathrm{CV}$ penetration rate from the macroscopic level. It cannot take the $\mathrm{CV}$ impact on the traffic flow into account from the microscopic level, such as lane-changing, acceleration, deceleration, etc. In this paper, the CV impact is considered only on the macroscopic level, which is intuitive and easy to implement. In future research, we will keep improving the model structure by taking more factors into account.

\subsection{Real-world implementation}

In this paper, the model is only implemented under simulation environment, which lacks the real-world data to calibrate the parameter. The parameter calibrating is the most challenging part of a traffic flow model's real applications, which can be solved in the future when more CVs drive on the road.

\section{Conclusions}

The purpose of this study is to study the CV impact on highway operational performance under a mixed $\mathrm{CV}$ and $\mathrm{RV}$ environment. As expected that CVs and RVs will co-exist on the transportation network in a long period. It is critical to understand how optimal speed control of CVs will affect the behaviors of nearby RVs. According to the different traffic characteristics between CVs and RVs, our research firstly analyzed how the CVs' implementation can affect highway capacity under both one-lane and multi-lane cases. A hypothesis was then made that there shall exist a critical CV penetration rate that can maximize the benefits of $\mathrm{CV}$ implementations. To prove this concept with numerical analysis, this study implemented a macroscopic mixed traffic flow model along with an embedded $\mathrm{CV}$ speed optimization function to simulate the mixed traffic pattern under various conditions. The simulation results revealed that performing optimal speed control to CVs will concurrently benefit RVs due to their interactions in the traffic streams. The highway capacity will consequently be increased. Also, given a demand pattern on a target freeway segment, there should exist a critical $\mathrm{CV}$ penetration rate that can significantly reduce the speed difference between RVs and CVs. With the increase of 
Figure 10 Time-dependent travel time under different levels of demand

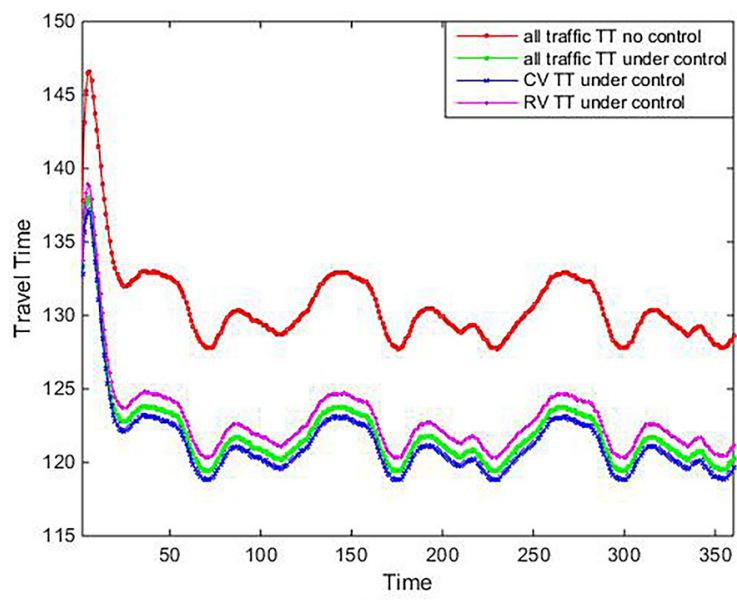

(a)

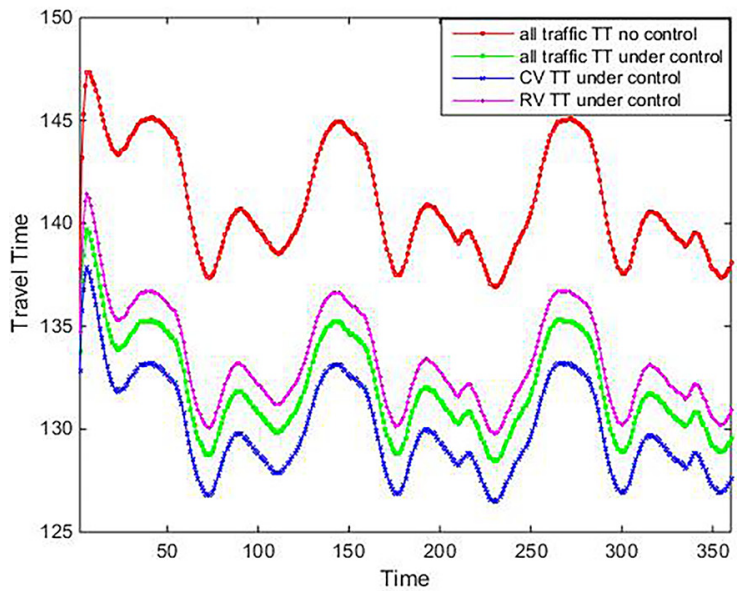

(c)

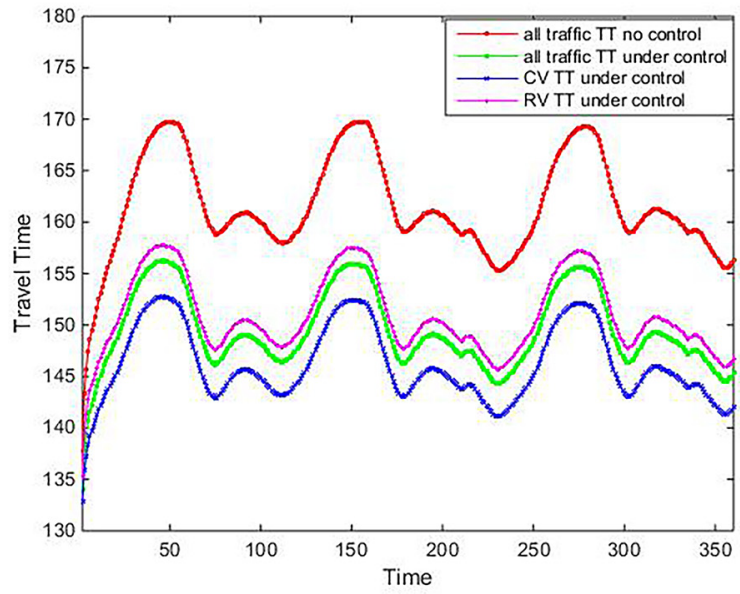

(e)

traffic demand volumes, such critical $\mathrm{CV}$ rate intends to be smaller on the same freeway sketch.

Future research directions include:

- develop theoretical methods to model the CV impact within the traffic stream;

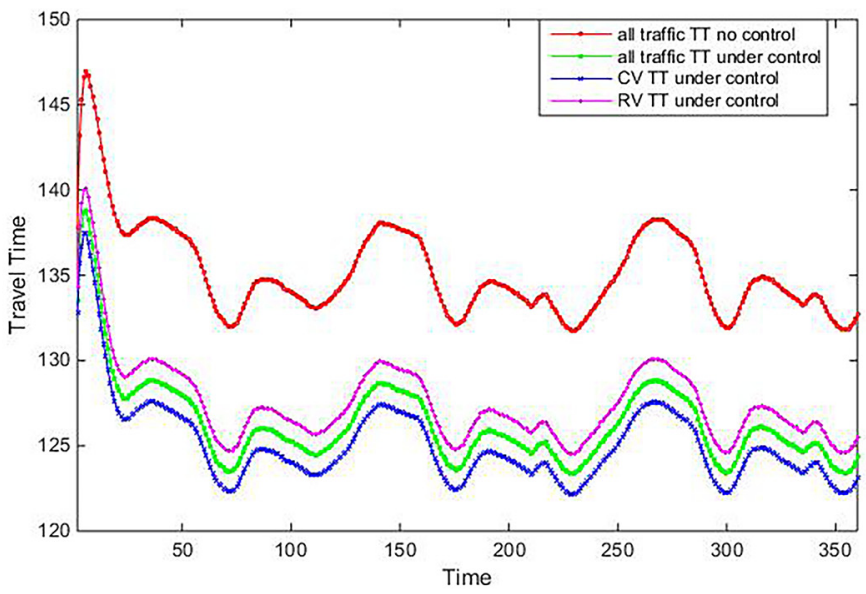

(b)

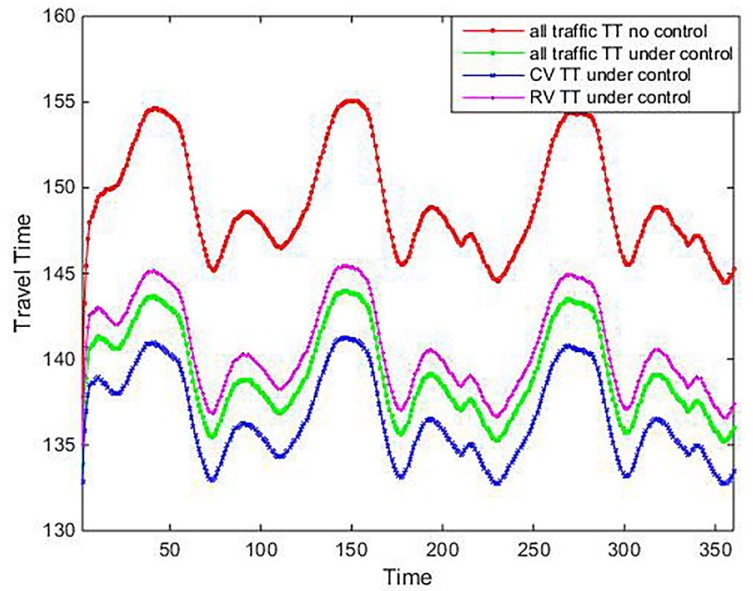

(d)

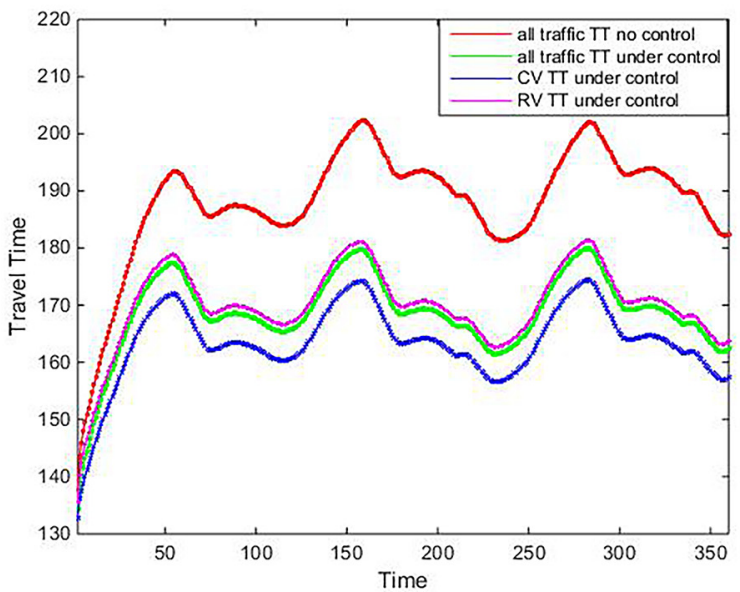

(f)

- prove the existence of critical CV penetration rate that can maximize the CV implementation benefits; and

- produce either close-formed equations or guidelines to estimate highway capacity under mixed $\mathrm{CV}$ and $\mathrm{RV}$ environment. 


\section{References}

Abdel-Aty, M., Dilmore, J. and Hsia, L. (2006), “Applying variable speed limits and the potential for crash migration", Transportation Research Record: Fournal of the Transportation Research Board, Vol. 1953 No. 1, pp. 21-30.

Abdel-Aty, M., Cunningham, R.J., Gayah, V.V. and Hsia, L. (2008), "Dynamic variable speed limit strategies for realtime crash risk reduction on freeways", Transportation Research Record: Fournal of the Transportation Research Board, Vol. 2078 No. 1, pp. 108-116.

Abdulsattar, H., Siam, M.R.K. and Wang, H. (2020), "Characterisation of the impacts of autonomous driving on highway capacity in a mixed traffic environment: an agentbased approach", IET Intelligent Transport Systems, Vol. 14 No. 9, pp. 1132-1141.

Ahn, S., Cassidy, M.J. and Laval, J. (2004), "Verification of a simplified car-following theory", Transportation Research Part B: Methodological, Vol. 38 No. 5, pp. 431-440.

Bansal, P. and Kockelman, K.M. (2017), "Forecasting Americans' long-term adoption of connected and autonomous vehicle technologies", Transportation Research Part A: Policy and Practice, pp. 49-63.

Carlson, R.C., Papamichail, I., Papageorgiou, M. and Messmer, A. (2010), "Optimal motorway traffic flow control involving variable speed limits and ramp metering", Transportation Science, Vol. 44 No. 2, pp. 238-253.

Chen, D., Ahn, S., Chitturi, M. and Noyce, D.A. (2017), "Towards vehicle automation: roadway capacity formulation for traffic mixed with regular and automated vehicles", Transportation Research Part B: Methodological, Vol. 100, pp. 196-221.

Chen, Z., He, F., Zhang, L. and Yin, Y. (2016), “Optimal deployment of autonomous vehicle lanes with endogenous market penetration", Transportation Research Part C: emerging Technologies, Vol. 72, pp. 143-156.

Ghiasi, A., Hussain, O., Qian, Z.S. and Li, X. (2017), “A mixed traffic capacity analysis and lane management model for connected automated vehicles: a Markov chain method", Transportation Research Part B: Methodological, Vol. 106, pp. 266-292.

Han, Y., Chen, D. and Ahn, S. (2017), "Variable speed limit control at fixed freeway bottlenecks using connected vehicles", Transportation Research Part B: Methodological, Vol. 98, pp. 113-134.

Hegyi, A., De Schutter, B. and Hellendoorn, J. (2005), "Optimal coordination of variable speed limits to suppress shock waves", IEEE Transactions on Intelligent Transportation Systems, Vol. 6 No No. 1, pp. 102-112.

Hu, J., Bhowmick, P., Arvin, F., Lanzon, A. and Lennox, B. (2020), "Cooperative control of heterogeneous connected vehicle platoons: an adaptive leader-following approach", IEEE Robotics and Automation Letters, Vol. 5 No. 2, pp. 977-984.

Hao, W. Liu, L. Yang, X. Li, Y. and Byon, Y.J. (2020), "Reducing CACC platoon disturbances caused by state jitters by combining two stages driving state recognition with multiple platoons' strategies and risk prediction", IEEE Transactions on Intelligent Transportation Systems.
Huang, K., Yang, X., Lu, Y., Mi, C.C. and Kondlapudi, P. (2018), "Ecological driving system for connected/automated vehicles using a two-stage control hierarchy", IEEE Transactions on Intelligent Transportation Systems, Vol. 19 No. 7, pp. 2373-2384.

Jia, D. and Ngoduy, D. (2016), "Platoon based cooperative driving model with consideration of realistic inter-vehicle communication", Transportation Research Part C: Emerging Technologies, Vol. 68, pp. 245-264.

Kang, K.P., Chang, G.L. and Zou, N. (2004), "Optimal dynamic speed-limit control for highway work zone operations", Transportation Research Record: Fournal of the Transportation Research Board, Vol. 1877 No. 1, pp. 77-84.

Khondaker, B. and Kattan, L. (2015), "Variable speed limit: a microscopic analysis in a connected vehicle environment", Transportation Research Part C: Emerging Technologies, Vol. 58, pp. 146-159.

Kwon, E., Brannan, D., Shouman, K., Isackson, C. and Arseneau, B. (2007), "Development and field evaluation of variable advisory speed limit system for work zones", Transportation Research Record: Fournal of the Transportation Research Board, Vol. 2015Vol. 2015 No. 1, pp. 12-18.

Liu, H., Wei, H., Zuo, T., Li, Z. and Yang, Y.J. (2017), "Finetuning ADAS algorithm parameters for optimizing traffic safety and mobility in connected vehicle environment", Transportation Research Part C: emerging Technologies, Vol. 76, pp. 132-149.

Lyu, P., Lin, Y., Wang, L. and Yang, X. (2017), "Variable speed limit control for delay and crash reductions at freeway work zone area", fournal of Transportation Engineering, Part A: Systems, Vol. 143 No. 12.

Lavasani, M., Jin, X. and Du, Y. (2016), "Market penetration model for autonomous vehicles on the basis of earlier technology adoption experience", Transportation Research Record: Fournal of the Transportation Research Board, Vol. 2597 No. 1, pp. 67-74.

Lu, N., Cheng, N., Zhang, N., Shen, X. and Mark, J.W. (2014), "Connected vehicles: solutions and challenges", IEEE Internet of Things fournal, Vol. 1 No. 4, pp. 289-299.

National Highway Traffic Safety Administration (NHTSA) (2016), "Vehicle-To-Vehicle communication technology for light vehicles", Office of Regulatory Analysis and Evaluation National Center for Statistics and Analysis, No. 150.

Newell, G.F. (2002), "A simplified car-following theory: a lower order model", Transportation Research Part B: Methodological, Vol. 36 No. 3, pp. 195-205.

Papageorgiou, M., Blosseville, J.M. and Hadj-Salem, H. (1990), "Modelling and real-time control of traffic flow on the Southern part of boulevard peripherique in Paris: part I: modelling", Transportation Research Part A: General, Vol. 24 No. 5, pp. 345-359.

Papageorgiou, M., Kosmatopoulos, E. and Papamichail, I. (2008), "Effects of variable speed limits on motorway traffic flow", Transportation Research Record: Fournal of the Transportation Research Board, Vol. 2047 No. 1, pp. 37-48.

Rahman, M.S. and Abdel-Aty, M. (2018), "Longitudinal safety evaluation of connected vehicles' platooning on expressways", Accident Analysis E Prevention, Vol. 117, pp. 381-391. 
Steel, P., McGregor, R.V., Guebert, A.A. and McGuire, T.M. (2005), "Application of variable speed limits along the trans Canada highway in banff national park", Annual Conference of the Transportation Association of Canada.

Talebpour, A. and Mahmassani, H.S. (2016), "Influence of connected and autonomous vehicles on traffic flow stability and throughput", Transportation Research Part C: Emerging Technologies, Vol. 71, pp. 143-163.

Ulfarsson, G.F., Shankar, V.N. and Vu, P. (2005), "The effect of variable message and speed limit signs on mean speeds and speed deviations", International fournal of Vehicle Information and Communication Systems, Vol. 1 Nos. 1/2, pp. 69-87.

Wan, N., Vahidi, A. and Luckow, A. (2016), “Optimal speed advisory for connected vehicles in arterial roads and the impact on mixed traffic", Transportation Research Part C: Emerging Technologies, Vol. 69, pp. 548-563.

Wang, Y. and Papageorgiou, M. (2005), "Real-time freeway traffic state estimation based on extended kalman filter: a general approach", Transportation Research Part B: Methodological, Vol. 39 No. 2, pp. 141-167.
Wang, Q., Yang, X., Huang, Z. and Yuan, Y. (2020), “Multivehicle trajectory design during cooperative adaptive cruise control platoon formation", Transportation Research Record: Fournal of the Transportation Research Board, Vol. 2674 No. 4, pp. 30-41.

Yang, H., Wang, Z. and Xie, K. (2017), "Impact of connected vehicles on mitigating secondary crash risk", International Fournal of Transportation Science and Technology, Vol. 6 No. 3, pp. 196-207.

Yang, X.T. Huang, K. Zhang, Z. Zhang, Z.A. and Lin, F. (2020), "Eco-Driving system for connected automated vehicles: multi-Objective trajectory optimization", IEEE Transactions on Intelligent Transportation Systems.

Yang, X., Lu, Y. and Lin, Y. (2017), “Optimal variable speed limit control system for freeway work zone operations", Fournal of Computing in Civil Engineering, Vol. 31 No. 1.

Zhang, Z. Yang, X. and X, L. (2018), "Freeway traffic optimal control with mixed connected vehicles and regular cars".

\section{Corresponding author}

Xianfeng Yang can be contacted at: x.yang@utah.edu 